\title{
Estimação da área foliar de mucuna cinza por meio de método não destrutivo
}

\author{
Leaf area estimation of velvet bean through non destructive method
}

\author{
Alberto Cargnelutti Filho ${ }^{\mathrm{I} *}$ Marcos Toebe ${ }^{\mathrm{II}}$ Cláudia Burin ${ }^{\mathrm{III}}$ André Luis Fick ${ }^{\mathrm{III}}$ \\ Ismael Mario Márcio Neu ${ }^{\text {IV }}$ Giovani Facco ${ }^{\text {IV }}$
}

\begin{abstract}
O objetivo deste trabalho foi modelar a área foliar RESUMO de mucuna cinza determinada por fotos digitais em função das dimensões do limbo do folíolo central da folha. Foi conduzido um experimento de $256 \mathrm{~m}^{2}$ com a cultura de mucuna cinza em área experimental da Universidade Federal de Santa Maria. Em seis períodos de desenvolvimento da cultura (29, 43, 57, 73, 87 e 101 dias após a emergência), foram coletadas, aleatoriamente, o total de 790 folhas. Cada folha é composta pelos folíolos esquerdo, central e direito. Nessas 790 folhas, foi mensurado o comprimento (CFC) e a largura ( $L F C)$ e calculado o produto do comprimento vezes a largura $(C F C \times L F C)$ do limbo do folíolo central. A seguir, determinouse a área foliar (soma da área foliar dos folíolos esquerdo, central e direito) por meio do método de fotos digitais $(Y)$. Posteriormente, separou-se, aleatoriamente, 650 folhas para a construção de modelos do tipo quadrático, potência e linear, de Y em função do CFC, da LFC, e/ou do CFC $\times L F C$, e 140 folhas para a validação dos modelos. Em mucuna cinza, o modelo potência $\left(\hat{Y}=3,6450 x^{1,9479}, R^{2}=0,9886\right)$ com base na largura do limbo do folíolo central é adequado para estimar a área foliar obtida por fotos digitais.
\end{abstract}

Palavras-chave: Stizolobium cinereum, foto digital, dimensão foliar, modelo linear, correlação.

\section{ABSTRACT}

The objective of this research was to model the leaf area of velvet bean determined by digital photos with the dimensions of the central leaflet limb of the leaf. A experiment of $256 \mathrm{~m}^{2}$ was conducted with the culture of velvet bean in the
Universidade Federal de Santa Maria. In six periods of culture development (29, 43, 57, 73, 87 and 101 days after emergence) were collected, randomly, 790 leaves. Each leaf is composed of the left, center and right leaflets. In these 790 leaves were measured the length (CFC) and width (LFC) and calculated the product length width $(C F C \times L F C)$ of the central leaflet. It was determined the leaf area (the leaf area sum of the left, center and right leaflets) by the method of digital photos $(Y)$. Later, 650 leaves were randomly separated, to build the quadratic model, potency and linear of $Y$ in function of the $C F C, L F C$ and/or CFC $\times$ LFC. It was used 140 leaves for validation. The potency model of leaf area obtained by method of digital photos $\left(\hat{Y}=3.6450 x^{1.9479}, R^{2}=0.9886\right)$ based on the width central leaflet, is adequate to estimate leaf area in velvet bean.

Key words: Stizolobium cinereum, digital photo, leaf dimension, linear model, correlation.

Plantas leguminosas apresentam elevada capacidade de fixação biológica de nitrogênio, sendo muito utilizadas na rotação de culturas. A mucuna cinza apresenta adaptabilidade a diferentes condições de solo e, nessa cultura, estudos têm sido realizados quanto à decomposição e liberação de nitrogênio em seus resíduos culturais (RIBAS et al., 2010).

A quantificação da área foliar de uma cultura é importante, pois está relacionada com a capacidade de absorção de radiação solar e com a transpiração. A

'Departamento de Fitotecnia, Centro de Ciências Rurais (CCR), Universidade Federal de Santa Maria (UFSM), 97105-900, Santa Maria, RS, Brasil. E-mail: cargnelutti@pq.cnpq.br. *Autor para correspondência.

"Programa de Pós-graduação em Agronomia, UFSM, Santa Maria, RS, Brasil.

IIICurso de Engenharia Florestal, UFSM, Santa Maria, RS, Brasil.

${ }^{\mathrm{IV}}$ Curso de Agronomia, UFSM, Santa Maria, RS, Brasil. 
área foliar pode ser determinada diretamente através do método de fotos digitais. Segundo ADAMI et al. (2008), o método de fotos digitais pode substituir o método LI-COR (método padrão), na estimação da área foliar, em folíolos íntegros e danificados de soja, com vantagens de custo inferior e acuracidade similar. Indiretamente, a área foliar pode ser obtida através de modelos de estimação da área foliar (obtida por fotos digitais, por exemplo) em função das dimensões lineares das folhas, conforme realizado na cultura de crambe (TOEBE et al., 2010).

O objetivo deste trabalho foi modelar a área foliar de mucuna cinza (Stizolobium cinereum), determinada por fotos digitais em função do comprimento, ou da largura e/ou do produto comprimento vezes largura do limbo do folíolo central da folha.

Foi conduzido um experimento de $256 \mathrm{~m}^{2}$ com a cultura de mucuna cinza na área experimental do Departamento de Fitotecnia da Universidade Federal de Santa Maria (latitude 2942'S, longitude 5349' W e altitude de $95 \mathrm{~m}$ ). $\mathrm{O}$ espaçamento foi de $0,5 \mathrm{~m}$ entre fileiras e $0,125 \mathrm{~m}$ entre plantas na fileira, totalizando 16 plantas por $\mathrm{m}^{2}$. A semeadura foi realizada no dia 13/11/2010 com adubação de base de $40 \mathrm{~kg} \mathrm{ha}^{-1} \mathrm{de} \mathrm{N}, 150 \mathrm{~kg} \mathrm{ha}^{-1}$ de $\mathrm{P}_{2} \mathrm{O}_{5}$ e $100 \mathrm{~kg} \mathrm{ha}^{-1}$ de $\mathrm{K}_{2} \mathrm{O}$. Foram coletadas, aleatoriamente, 90 folhas, aos 29 dias após a emergência (DAE), e 140 folhas em outros cinco períodos (43, 57, 73, 87 e 101DAE), totalizando 790 folhas compostas de três folíolos (esquerdo, central e direito).

Nessas 790 folhas, foi mensurado o comprimento (CFC) e a largura (LFC) do limbo do folíolo central, com régua milimetrada. Após, foi calculado o produto comprimento vezes largura $(\mathrm{CFC} \times \mathrm{LFC})$ do limbo do folíolo central. As mesmas folhas (compostas de três folíolos) foram fotografadas sobre papel milimetrado por meio de uma câmera digital da marca Sony, modelo DSC-W110. Essas imagens (fotos) foram processadas com o programa Sigma Scan Pro v. 5.0 (JANDEL SCIENTIFIC, 1991), para a determinação da área foliar (soma da área foliar dos folíolos esquerdo, central e direito) por meio do método de fotos digitais (Y).

Posteriormente, entre as 790 folhas, foram selecionadas, aleatoriamente, 140 folhas que foram utilizadas apenas na validação dos modelos. Com os dados do CFC, da LFC, do CFC $\times$ LFC e de Y das 650 folhas restantes, foram calculadas medidas de tendência central, de variabilidade, de assimetria e de curtose e verificada a normalidade, por meio do teste de Kolmogorov-Smirnov. Após, com os dados dessas 650 folhas, modelou-se a área foliar determinada por fotos digitais (Y) em função do CFC, da LFC e do CFC $\times$ LFC, por meio dos modelos: quadrático
$\left(\mathrm{Y}=\mathrm{a}+\mathrm{bx}+\mathrm{cx}^{2}+\mathrm{e}\right)$, potência $\left(\mathrm{Y}=\mathrm{ax} \mathrm{x}^{\mathrm{b}}+\mathrm{e}\right)$ e linear $(\mathrm{Y}=\mathrm{a}+\mathrm{bx}+\mathrm{e})$, totalizando nove modelos (três modelos $\times$ três variáveis independentes), nos quais $\mathrm{x}$ representa as dimensões foliares e o termo erro de cada modelo é representado pela letra e. Nesses modelos, assume-se que as dimensões foliares foram mensuradas livres de erros de medida.

A validação dos nove modelos de estimação de área foliar foi realizada com base nos 140 valores estimados pelo modelo $\left(\hat{\mathrm{Y}}_{\mathrm{i}}\right)$ e os 140 valores observados $\left(\mathrm{Y}_{\mathrm{i}}\right)$. Em cada modelo, foi ajustada uma regressão linear simples $\left(\hat{\mathrm{Y}}_{\mathrm{i}}=\mathrm{a}+\mathrm{b} \mathrm{Y}_{\mathrm{i}}\right)$ da área foliar estimada pelo modelo (variável dependente) em função da área foliar observada (variável independente). Foram testadas as hipóteses $\mathrm{H}_{0}$ : $\mathrm{a}=0$ versus $\mathrm{H}_{1}$ : $\mathrm{a} \neq 0$ e $\mathrm{H}_{0}$ : $\mathrm{b}=1$ versus $\mathrm{H}_{1}: \mathrm{b} \neq 1$, por meio do teste $\mathrm{t}$ de Student a $5 \%$ de probabilidade de erro. A seguir, foram calculados os coeficientes de correlação linear de Pearson (r) e de determinação $\left(R^{2}\right)$ entre $\hat{Y}_{i}$ e $Y_{i}$. Para cada modelo, foi calculado o erro absoluto médio (EAM), a raiz do quadrado médio do erro (RQME) e o índice de Willmott (WILLMOTT, 1981), por meio, respectivamente, das

expressões $E A M=\frac{\sum_{i=1}^{n}\left|\hat{Y}_{i}-Y_{i}\right|}{n}, R Q M E=\sqrt{\frac{\sum_{i=1}^{n}\left(\hat{Y}_{i}-Y_{i}\right)^{2}}{n}}$ e $d=1-\left[\frac{\sum_{i=1}^{n}\left(\hat{Y}_{i}-Y_{i}\right)^{2}}{\sum_{i=1}^{n}\left(\left|\hat{Y}_{i}-\bar{Y}\right|+\left|Y_{i}-\bar{Y}\right|\right)^{2}}\right]$, em que $\hat{Y}_{i}$ são os valores estimados de área foliar, $\mathrm{Y}_{\mathrm{i}}$ os valores observados de área foliar por meio do método de fotos digitais, $\bar{Y}$ é a média dos valores observados e n é o número de folhas $(n=140)$.

Os modelos que melhor estimam a área foliar de mucuna cinza em função do CFC, ou da LFC e/ou do CFC $\times$ LFC do limbo do folíolo central são aqueles que apresentam: coeficiente linear não diferente de zero, coeficiente angular não diferente de um, coeficientes de correlação linear de Pearson e de determinação mais próximos de um, erro absoluto médio e raiz do quadrado médio do erro mais próximo de zero, e índice d de Willmott mais próximo de um. As análises estatísticas foram realizadas com o auxílio do aplicativo Office Excel. Em média, as 650 folhas utilizadas na construção dos modelos de estimação de área foliar apresentaram folíolo central com limbo de 12,554 e 9,599cm de comprimento e largura, respectivamente, e área foliar (soma da área foliar dos folíolos esquerdo, central e direito) de $326,227 \mathrm{~cm}^{2}$ (Tabela 1). De maneira geral, o CFC e a LFC apresentaram CV próximos a 30\% e o $\mathrm{CFC} \times \mathrm{LFC}$ e Y próximos a 55\%. Essa ampla variabilidade dos dados é importante na construção de modelos, pois permite a utilização destes para folhas de diferentes tamanhos e mensuradas em diferentes períodos de desenvolvimento da cultura. 
Tabela 1 - Número de folhas (n), mínimo, máximo, média, coeficiente de variação (CV), variância, curtose, assimetria e p-valor do teste de kolmogorov-Smirnov do comprimento (CFC), da largura (LFC), do produto comprimento vezes largura (CFC $\times$ LFC) do limbo do folíolo central e da área foliar (soma da área foliar dos folíolos esquerdo, central e direito), determinada por fotos digitais (Y) de 650 folhas de mucuna cinza (Stizolobium cinereum) coletadas em seis períodos.

\begin{tabular}{|c|c|c|c|c|c|c|c|c|c|}
\hline & $\mathrm{n}$ & Mínimo & Máximo & Média & CV(\%) & Variância & Curtose $^{(1)}$ & Assimetria $^{(2)}$ & P-valor \\
\hline & \multicolumn{9}{|c|}{29 dias após a emergência } \\
\hline CFC & 76 & 4,400 & 15,100 & 10,383 & 19,098 & 3,932 & $3,694^{\mathrm{ns}}$ & $-0,314^{*}$ & 0,788 \\
\hline LFC & 76 & 2,800 & 11,500 & 7,576 & 21,605 & 2,679 & $3,602^{\mathrm{ns}}$ & $-0,085^{\mathrm{ns}}$ & 0,891 \\
\hline $\mathrm{CFC} \times \mathrm{LFC}$ & 76 & 12,320 & 173,650 & 81,787 & 38,608 & 997,090 & $3,632^{\mathrm{ns}}$ & $0,540 *$ & 0,490 \\
\hline \multirow[t]{2}{*}{$\mathrm{Y}$} & 76 & 25,864 & 413,445 & 188,554 & 38,606 & $5.298,875$ & $4,047^{\mathrm{ns}}$ & $0,556^{*}$ & 0,170 \\
\hline & \multicolumn{9}{|c|}{43 dias após a emergência } \\
\hline CFC & 110 & 4,400 & 16,200 & 12,477 & 20,098 & 6,289 & $3,930 *$ & $-1,114^{*}$ & 0,063 \\
\hline LFC & 110 & 3,200 & 12,100 & 8,930 & 21,824 & 3,798 & $3,600^{\text {ns }}$ & $-0,960 *$ & 0,129 \\
\hline $\mathrm{CFC} \times \mathrm{LFC}$ & 110 & 14,080 & 196,020 & 116,136 & 35,994 & $1.747,364$ & $2,640^{\mathrm{ns}}$ & $-0,529 *$ & 0,263 \\
\hline \multirow[t]{2}{*}{ Y } & 110 & 33,970 & 506,419 & 276,657 & 35,638 & $9.721,020$ & $2,778^{\text {ns }}$ & $-0,512^{*}$ & 0,201 \\
\hline & \multicolumn{9}{|c|}{57 dias após a emergência } \\
\hline CFC & 113 & 3,800 & 19,100 & 12,387 & 34,464 & 18,224 & $2,030 *$ & $-0,470 *$ & 0,111 \\
\hline LFC & 113 & 2,900 & 14,500 & 9,399 & 33,872 & 10,135 & $2,058^{*}$ & $-0,437^{\mathrm{ns}}$ & 0,196 \\
\hline $\mathrm{CFC} \times \mathrm{LFC}$ & 113 & 11,020 & 276,950 & 129,794 & 57,240 & $5.519,593$ & $1,785^{*}$ & $-0,016^{\mathrm{ns}}$ & 0,241 \\
\hline \multirow[t]{2}{*}{$\mathrm{Y}$} & 113 & 26,684 & 695,070 & 326,033 & 57,612 & $35.281,609$ & $1,806^{*}$ & $0,008^{\text {ns }}$ & 0,322 \\
\hline & \multicolumn{9}{|c|}{73 dias após a emergência } \\
\hline CFC & 114 & 4,300 & 20,200 & 12,721 & 32,358 & 16,944 & $2,196^{\mathrm{ns}}$ & $-0,465^{*}$ & 0,200 \\
\hline LFC & 114 & 3,000 & 16,300 & 9,953 & 34,098 & 11,517 & $2,199^{\mathrm{ns}}$ & $-0,429^{\mathrm{ns}}$ & 0,106 \\
\hline $\mathrm{CFC} \times \mathrm{LFC}$ & 114 & 12,900 & 317,140 & 140,275 & 56,135 & $6.200,499$ & $2,111^{*}$ & $0,069^{\text {ns }}$ & 0,308 \\
\hline \multirow[t]{2}{*}{$\mathrm{Y}$} & 114 & 31,845 & 774,162 & 351,310 & 56,014 & $38.722,890$ & $2,096^{*}$ & $0,067^{\text {ns }}$ & 0,353 \\
\hline & \multicolumn{9}{|c|}{87 dias após a emergência } \\
\hline CFC & 119 & 4,300 & 19,300 & 13,166 & 28,183 & 13,767 & $2,495^{\mathrm{ns}}$ & $-0,531^{*}$ & 0,284 \\
\hline LFC & 119 & 3,100 & 16,200 & 10,401 & 30,221 & 9,880 & $2,289^{\mathrm{ns}}$ & $-0,419^{\mathrm{ns}}$ & 0,214 \\
\hline $\mathrm{CFC} \times \mathrm{LFC}$ & 119 & 13,330 & 312,660 & 148,345 & 50,671 & $5.650,256$ & $2,012^{*}$ & $0,021^{\mathrm{ns}}$ & 0,562 \\
\hline \multirow[t]{2}{*}{$\mathrm{Y}$} & 119 & 28,909 & 754,960 & 374,266 & 50,900 & $36.291,331$ & $1,989 *$ & $-0,006^{\mathrm{ns}}$ & 0,574 \\
\hline & \multicolumn{9}{|c|}{101 dias após a emergência } \\
\hline CFC & 118 & 4,700 & 20,200 & 13,404 & 28,836 & 14,940 & $2,277^{\mathrm{ns}}$ & $-0,377^{\mathrm{ns}}$ & 0,275 \\
\hline LFC & 118 & 3,700 & 16,600 & 10,568 & 31,191 & 10,865 & $2,129 *$ & $-0,224^{\mathrm{ns}}$ & 0,328 \\
\hline $\mathrm{CFC} \times \mathrm{LFC}$ & 118 & 18,330 & 330,340 & 154,105 & 53,124 & $6.702,248$ & $2,176^{\mathrm{ns}}$ & $0,193^{\mathrm{ns}}$ & 0,713 \\
\hline \multirow[t]{2}{*}{ Y } & 118 & 47,386 & 824,461 & 388,612 & 51,718 & $40.394,368$ & $2,164^{\mathrm{ns}}$ & $0,126^{\mathrm{ns}}$ & 0,461 \\
\hline & \multicolumn{9}{|c|}{ Todas as coletas } \\
\hline CFC & 650 & 3,800 & 20,200 & 12,554 & 29,338 & 13,565 & $2,406 *$ & $-0,331^{*}$ & 0,012 \\
\hline LFC & 650 & 2,800 & 16,600 & 9,599 & 31,819 & 9,329 & $2,323 *$ & $-0,107^{\mathrm{ns}}$ & 0,215 \\
\hline $\mathrm{CFC} \times \mathrm{LFC}$ & 650 & 11,020 & 330,340 & 131,517 & 54,644 & $5.164,783$ & $2,434^{*}$ & $0,365^{*}$ & 0,024 \\
\hline $\mathrm{Y}$ & 650 & 25,864 & 824,461 & 326,227 & 55,545 & $32.833,794$ & $2,382 *$ & $0,384 *$ & 0,009 \\
\hline
\end{tabular}

(1) * Curtose difere de três, pelo teste t, em nível de $5 \%$ de probabilidade de erro. ${ }^{\text {ns }}$ Não-significativo.

(2) * Assimetria difere de zero, pelo teste t, em nível de 5\% de probabilidade de erro. ${ }^{\text {ns }}$ Não-significativo.

Em relação ao CFC, LFC, CFC $\times$ LFC e Y das coletas realizadas em seis períodos, em $41,7 \%$ dos casos, verificaram-se curtose diferente de três e assimetria diferente de zero $(\mathrm{P} \leq 0,05)$. Contudo, todos os dados ajustaram-se à distribuição normal, de acordo com os p-valores $(\mathrm{P}>0,05)$ do teste de KolmogorovSmirnov. Ao considerar todas as folhas coletadas (650), apenas a LFC apresentou assimetria não diferente de zero e distribuição normal. O elevado número de folhas
(650) contribuiu para que pequenos desvios das estimativas em relação à hipótese de nulidade da curtose, da assimetria e da normalidade fossem significativos. Segundo LEVINE et al. (2008), quando o número de observações é elevado (maior que 30), com base no teorema do limite central, a distribuição da média amostral se ajusta à distribuição normal. Assim, pode-se inferir boa aderência dos dados à distribuição normal. 
De maneira geral, os modelos quadrático, potência e linear, que relacionam CFC, LFC e CFC ×LFC com a área foliar determinada por fotos digitais (Y), apresentaram elevados coeficientes de determinação $\left(\mathrm{R}^{2} \geq 0,9299\right)$ (Tabela 2), sugerindo a utilização de qualquer um dos nove modelos para estimar a área foliar de mucuna cinza. Em relação as três variáveis independentes, os melhores modelos foram obtidos com o CFC $\times$ LFC $\left(R^{2} \geq 0,9880\right)$, seguido dos modelos construídos a partir da LFC $\left(\mathrm{R}^{2} \geq 0,9616\right)$. Em crambe, TOEBE et al. (2010) também verificaram melhores ajustes dos modelos obtidos a partir da largura em relação ao comprimento. Quanto ao tipo de modelo utilizado, maior precisão foi observada nos modelos potência $\left(R^{2} \geq 0,9805\right)$ e quadrático $\left(R^{2} \geq 0,9702\right)$, concordando com TOEBE et al. (2010).

Com base nos indicadores usados para a validação dos modelos, houve maior precisão dos modelos que utilizam o CFC×LFC na estimação da área foliar, com coeficiente linear não diferente de zero, coeficientes de correlação linear de Pearson e de determinação mais próximos de um, menor erro absoluto médio e raiz do quadrado médio do erro e índices d de Willmott mais próximo de um, embora o modelo tipo potência apresente coeficiente angular diferente de um $(\mathrm{P} \leq 0,05)$ (Tabela 2). O modelo tipo potência em função da LFC $\left(\hat{Y}=3,6450 x^{1,9479}\right)$ é o único dos seis modelos restantes que apresentou indicadores de validação semelhantes aos encontrados para os modelos construídos com base no CFC $\times$ LFC. Portanto, este modelo deve, preferencialmente, ser adotado em função de possibilitar a estimação da área foliar (soma da área foliar dos folíolos esquerdo, central e direito) de mucuna cinza com elevada precisão, necessitando apenas da mensuração da largura do limbo do folíolo central.

Tabela 2 - Modelos para a determinação da área foliar obtida por fotos digitais (Y), utilizando o comprimento (CFC), a largura (LFC) e o produto comprimento vezes largura $(\mathrm{CFC} \times \mathrm{LFC})$ do limbo do folíolo central como variáveis independentes (x) e coeficiente de determinação ( $\mathrm{R}^{2}$ ) com base em 650 folhas. Validação dos modelos com base em coeficientes linear (a), angular (b), de correlação linear de Pearson (r) e de determinação $\left(R^{2}\right)$, obtidos na regressão linear ajustada entre a área foliar estimada (variável dependente) e a observada (variável independente). Erro absoluto médio (EAM), raiz do quadrado médio do erro (RQME) e índice d de Willmott calculados com base nas áreas foliares observadas e estimadas de 140 folhas de mucuna cinza (Stizolobium cinereum).

\begin{tabular}{|c|c|c|c|c|c|c|c|c|}
\hline Modelo & \multicolumn{2}{|c|}{ Variável independente (x) } & \multicolumn{3}{|c|}{ Equação } & \multicolumn{3}{|c|}{ Coeficiente de determinação } \\
\hline 1) Quadrático & \multicolumn{2}{|l|}{ CFC } & \multicolumn{3}{|c|}{$Y=34,7963-8,9814 x+2,3617 x^{2}$} & \multicolumn{3}{|c|}{0,9702} \\
\hline 2) Quadrático & \multicolumn{2}{|l|}{ LFC } & \multicolumn{3}{|c|}{$Y=-41,8370+13,0967 x+2,3886 x^{2}$} & \multicolumn{3}{|c|}{0,9813} \\
\hline 3) Quadrático & \multicolumn{2}{|c|}{$\mathrm{CFC} \times \mathrm{LFC}$} & \multicolumn{3}{|c|}{$Y=-2,7374+2,4943 x+0,00004 x^{2}$} & \multicolumn{3}{|c|}{0,9880} \\
\hline 4) Potência & \multicolumn{2}{|l|}{ CFC } & \multicolumn{3}{|c|}{$Y=1,6225 x^{2,0579}$} & \multicolumn{3}{|c|}{0,9805} \\
\hline 5) Potência & \multicolumn{2}{|l|}{ LFC } & \multicolumn{3}{|c|}{$Y=3,6450 x^{1,9479}$} & \multicolumn{3}{|c|}{0,9886} \\
\hline 6) Potência & \multicolumn{2}{|c|}{$\mathrm{CFC} \times \mathrm{LFC}$} & \multicolumn{3}{|c|}{$Y=2,3735 x^{1,0083}$} & \multicolumn{3}{|c|}{0,9921} \\
\hline 7) Linear & \multicolumn{2}{|c|}{ CFC } & \multicolumn{3}{|c|}{$Y=-269,3563+47,4429 x$} & \multicolumn{3}{|c|}{0,9299} \\
\hline 8) Linear & \multicolumn{2}{|l|}{ LFC } & \multicolumn{3}{|c|}{$Y=-232,1965+58,1737 x$} & \multicolumn{3}{|c|}{0,9616} \\
\hline 9) Linear & \multicolumn{2}{|c|}{$\mathrm{CFC} \times \mathrm{LFC}$} & \multicolumn{3}{|c|}{$Y=-3,3734+2,5061 x$} & \multicolumn{3}{|c|}{0,9880} \\
\hline Modelo & $\mathrm{x}$ & $a^{(1)}$ & $b^{(2)}$ & $r^{(3)}$ & $\mathrm{R}^{2}$ & EAM & RQME & d \\
\hline 1) Quadrático & CFC & $9,135^{*}$ & $0,975^{*}$ & $0,989 *$ & 0,980 & 21,602 & 27,405 & 0,995 \\
\hline 2) Quadrático & LFC & $7,172 *$ & $0,975^{*}$ & 0,993* & 0,986 & 16,157 & 22,538 & 0,996 \\
\hline 3) Quadrático & $\mathrm{CFC} \times \mathrm{LFC}$ & $4,075^{\mathrm{ns}}$ & $0,988^{\mathrm{ns}}$ & $0,995 *$ & 0,992 & 13,294 & 17,636 & 0,998 \\
\hline 4) Potência & CFC & $17,295^{*}$ & $0,941^{*}$ & 0,989* & 0,979 & 21,923 & 28,768 & 0,994 \\
\hline 5) Potência & LFC & $-1,907^{\mathrm{ns}}$ & $1,005^{\text {ns }}$ & $0,992 *$ & 0,986 & 16,401 & 23,266 & 0,996 \\
\hline 6) Potência & $\mathrm{CFC} \times \mathrm{LFC}$ & $5,169^{\mathrm{ns}}$ & $0,982 *$ & 0,995* & 0,992 & 13,288 & 17,691 & 0,998 \\
\hline 7) Linear & CFC & $28,132 *$ & $0,909 *$ & $0,967^{*}$ & 0,936 & 37,070 & 48,829 & 0,983 \\
\hline 8) Linear & LFC & $16,345^{*}$ & $0,940 *$ & $0,981^{*}$ & 0,964 & 28,014 & 36,798 & 0,990 \\
\hline 9) Linear & $\mathrm{CFC} \times \mathrm{LFC}$ & $4,111^{\mathrm{ns}}$ & $0,988^{\mathrm{ns}}$ & $0,995^{*}$ & 0,992 & 13,309 & 17,643 & 0,998 \\
\hline
\end{tabular}

(1) * Coeficiente linear difere de zero, pelo teste t, em nível de $5 \%$ de probabilidade de erro. ${ }^{\text {ns }}$ Não-significativo.

(2) * Coeficiente angular difere de um, pelo teste t, em nível de $5 \%$ de probabilidade de erro. ${ }^{\text {ns }}$ Não-significativo.

(3) * Coeficiente de correlação difere de zero, pelo teste t, em nível de $5 \%$ de probabilidade de erro. ${ }^{\text {ns }}$ Não-significativo. 


\section{REFERÊNCIAS}

ADAMI, M. et al. Estimativa de área de folíolos de soja usando imagens digitais e dimensões foliares. Bragantia, v.67, p.10531058, 2008. Disponível em: < http://www.scielo.br/pdf/brag/ v67n4/30.pdf >. Acesso em: 02 maio, 2011. doi: 10.1590/ S0006-87052008000400030.

JANDEL SCIENTIFIC. User's manual. California: Jandel Scientific, 1991. 280p.

LEVINE, D.M. et al. Estatística - teoria e aplicações. 5.ed. Rio de Janeiro: LTC, 2008. 752p.
RIBAS, R.G.T. et al. Decomposição, liberação e volatilização de nitrogênio em resíduos culturais de mucuna-cinza (Mucuna cinerea). Ciência e Agrotecnologia, v.34, p.878-885, 2010. Disponível em: < http://www.scielo.br/pdf/cagro/v34n4/ v34n4a12.pdf >. Acesso em: 02 maio, 2011. doi: 10.1590/ S1413-70542010000400012.

TOEBE, M. et al. Estimativa da área foliar de Crambe abyssinica por discos foliares e por fotos digitais. Ciência Rural, v.40, p.445-448, 2010. Disponível em: <http:// www.scielo.br/pdf/cr/v40n2/a475cr2110.pdf>. Acesso em: 02 maio, 2011. doi: 10.1590/S0103-84782010000200036.

WILlMOTT, C.J. On the validation of models. Physical Geography, v.2, p.184-194, 1981. 